\title{
Concussion: Implementing a Trauma Prevention Program in the Community
}

Jung $\mathrm{D}^{*}$

California State University, Fullerton, USA

*Corresponding author: Deanna Jung, DNP, APRN, AGACNP-BC, ACCNS-AG

Review Article

Volume 1 Issue 6

Received Date: September 18, 2017

Published Date: November 03, 2017 Coordinator, Pre-Licensure Programs and Assistant Professor, California State University, Fullerton, 800 N. State College Ave Fullerton, CA 92831, USA, Tel: 657-278-5365; E-mail: dejung@fullerton.edu

\section{Abstract}

Introduction: There are approximately 3.8 million concussions that occur annually. Sports injuries are the second leading cause of traumatic brain injuries among adolescents. Concussions represent approximately nine percent of all high school sports injuries. However, concussions are grossly underreported as it affects athletes playing time and possible scholarships they may be contending for. Underreporting of concussions can lead to long-term sequelae and gross deficits.

Aim: To identify a model, which will allow for implementation of an educational trauma prevention program in the community and can be used throughout the state of California to decrease the number of concussions, which occur annually while simultaneously decreasing morbidity and mortality rates of those injured?

Methods: Review how a clinical nurse specialist, an advanced practice nurse, can positively affect outcomes of concussed patients. Review how the PDCA model can be integrated in the process of such a program for the community to improve outcomes. Utilized the Cochrane Library and Cinhal Repositories for review of peer-reviewed articles including articles from the United States and ones in the English language.

Conclusion: The implementation of an educational trauma prevention program with the PDCA model by a CNS will decrease morbidity and mortality rates. The use of interdisciplinary teams is necessary to improve outcomes for concussed patients, which involves both the community as well as healthcare organizations alike.

Keywords: Concussion; Prevention; CNS; Traumatic; Community

\section{Introduction}

There are approximately 3.8 million concussions that occur annually [1]. Sports injuries are the second leading cause of traumatic brain injuries among adolescents and the number of concussions has continued to steadily rise over the past 10 years [1-3]. Concussions represent approximately nine percent of all high school sports injuries [1]. However, concussions are grossly underreported as it affects athletes playing time and possible scholarships they may be contending for [1]. Underreporting of concussions can lead to long term sequelae and gross deficits [1]. Concussions occur 


\section{Nursing \& Healthcare International Journal}

A concussion is defined as a complex pathophysiological process that affects the brain by an induced traumatic biomechanical force or rapid rotational acceleration that disrupt brain function $[1,4]$. Functional neuropathologic changes may occur rapidly and subside spontaneously, resulting in a normal diagnostic test result [1]. Risk factors associated with concussion in children include collision sports, history of prior concussions, vulnerable neck musculature, brain water content, vasculature, and amount of myelination which may lead to cerebral swelling associated with second impact syndrome $[1,5]$.

Symptoms which occur commonly with concussions include: headache, nausea, vomiting, confusion, dizziness or balance problems, poor concentration, sensitivity to light and/or noise, memory problems and emotional liability [6]. Cognitive effects from sustaining a concussion include: attenuated processing speed, memory impairment, and blunted reaction times that occur immediately and may improve spontaneously or remain persistent over time [4]. The importance of recognizing concussion symptoms and implementation of concussion management plans are evident in the passing of Assembly Bill 25 (AB25) into law [5].

Compliance involving concussion assessment, diagnosis, and management are now required under AB25 [7]. The recently implemented law, $A B 25$, requires student athletes suspected of sustaining a concussion be removed from the activity immediately and requires further evaluation by a health care professional for a graduated return to physical and mental activity [7]. Assembly Bill 25 also requires annual signatures from both athletes and parents on a concussion and head injury information sheet prior to participation in any sport [7]. Schools in which sports are offered are required to have a concussion management plan in place [7].

\section{Aim}

The knowledge focused evidence based practice project involves asking the following PICO question. In high school administration (coaches, athletic trainers, faculty, and school nurses) what is the effect of trauma prevention concussion education programs compared with no education on increased awareness, early identification of concussion symptoms with initiation of earlier treatment, decreased long term neurological complications, and decreased incidence of second impact syndrome occurring. The aim is to implement a trauma prevention program by an advanced practice nurse to decrease the number of concussions in young adult athletes in a community setting.

\section{Review of Literature}

Covassin, et al. (2012) [8] concluded educating coaches via the CDC's "Heads Up Concussion in Youth Sports" initiative increased the awareness of concussive symptoms, and had positive outcomes on management of concussive injuries. There was a trend in concussive incidence during high school sports from 8\%-27\% annually between 1997 through 2008 [5]. Lincoln and colleagues (2011) [5] found among twelve high school sports including both female and male participation repeat concussions represented $11 \%$ of all concussions reported increasing the risk of second impact syndrome. Meehan and colleagues (2012) [4] discovered with repeated mild concussions during vulnerable periods permanent cognitive deficits remained evident. The Centers for Disease Control (n.d.) [6] concur with Meehan, et al. (2012) [4] stating children from birth to 19 years of age are slower to recover from concussions than adults. Shenouda, et al. (2012) [9] found upon implementation of legislation on concussion management there were gaps in knowledge about symptoms of concussions and guidelines for return to play. There is a need for increased education and educational programs targeting coaches, parents, and school administrators due to the significant variability in practice [9]. Vagnozzi, et al. (2010) [10] concur with Shenouda, et al. (2012) [9] in regards to the unclear criteria for a safe return to play for athletes. The incidence of recurrent concussion, although mild, in an athlete increases three fold after the initial injury [10]. There is a significant need for a unanimously approved diagnostic approach to care for and manage newly and previously concussed athletes [10].

As research has shown there is an identified gap in knowledge of concussion symptoms and management [9]. The outcomes hoped to achieve are divided into three categories knowledge, process and practice change, and financial. Knowledge based outcomes include increasing awareness of concussive symptoms, management, and the importance of early identification and treatment in order to decrease long-term deficits [11]. Process change should begin during preseason [12]. Baseline cognitive testing would be required prior to initiation of sport play [12]. Sideline assessments, sports concussion assessment tool 2 (SCAT 2) or immediate post-concussion assessment and cognitive testing (ImPACT), would be completed when a concussion is suspected, with the athlete being removed from play for the remainder of the day [13,14]. Implementing an algorithm for return to school and play 


\section{Nursing \& Healthcare International Journal}

would be initiated with the appropriate referrals to include: a concussion specialist, rehabilitation, neuropsychologist, neurologist, physical therapist, or pediatrician [15-18]. Financial concerns for implementation of such a process change would include the cost of ImPACT or SCAT 2 testing, cost of rehabilitation, referrals to specialists such as a neuropsychologist, days missed from school, decline in academic progress, and cost of additional care for complex, or second impact syndrome from repetitive concussions, as well as legal implications $[11,15,17]$.

Implementation of a trauma prevention concussion education program in the community would include two conferences with the first targeted to coaches, athletic trainers, school nurses, and school faculty and administrators [1,8,11]. Additional conferences would target parents and athletes [11]. The conferences would include both north and south Orange County. The conferences would include a didactic portion as well as a return demonstration of the SCAT 2 and ImPACT testing. Business cards for referrals to specialists would be given during the conferences. A "playbook" educational pamphlet would be distributed to all in attendance as a tool to take back to their perspective schools.

\section{Methods}

During implementation of a trauma prevention education program for the community the PDCA model is used simultaneously [19]. The PDCA model is circular and constant which allows review of implementation measures and the ability to improve as needed [17]. During the plan phase the clinical nurse specialist (CNS) collects data via literature searches, investigating current practices and guidelines, research, and performing a systematic review. An effective and sustainable process change should be based on evidence from research [20].

The next phase in the PDCA model is the do phase. During this phase the CNS continues to collect and analyze data, and execute a plan to implement the process change. At this time the CNS would meet with a nationally recognized concussion expert to obtain insight on current practices and programs. Formulating and coordination of schedules to meet with trauma surgeons, emergency room physicians, the trauma coordinator, neurosurgeons physical therapists, school nurses, athletic trainers, neurologists, neuropsychologist, public relations and media department, and pediatricians at both a level II trauma center in south orange county as well as a pediatric hospital in Orange County would be coordinated by the CNS as well. The CNS would be responsible for distributing and writing the educational material to be given at the conferences and editing of changes. Next the CNS would identify sites where the conferences could be held. The CNS would also secure funds by donation from contributors to offset costs from the hospital. Lastly, the CNS would coordinate and facilitate nationally known expert panel speakers to speak at the conferences.

Once the conference has concluded the CNS would be in the check phase of the PDCA model, checking the progress of the process change at the high school level [19]. If coaches and athletic trainers were having difficulty implementing the process, review of obstacles and input would be necessary from the CNS. Review of the process improvement would be performed by the CNS and adjustments made where indicated. The importance of recognition of concussive symptoms and adhering to a management plan for athletes to return to play has been identified through various research $[1,4-10,20]$.

The act phase of the PDCA model is the ability to sustain the process change and also includes flexibility to make changes where necessary [20]. The PDCA model is a continuous model that improves quality process change [19]. The CNS would make needed adjustments as necessary. It is also important to note that if the process change were not working during this phase the PDCA model would begin altogether [19]. The adherence to AB25 would require sustainability for all athletes playing sports [6].

\section{Discussion}

The PDCA model has many positive aspects, as there is the ability to quickly review the processes, which have been implemented. It allows the CNS to determine whether the interventions are successful and also whether there is a need for the interventions to be augmented to better facilitate and improve the process change. The ability to make changes in a timely manner ultimately would improve outcomes of patients.

Additionally, the CNS is specifically trained to develop, write, and implement policies at different administrative levels. This allows the CNS to discuss, prepare, and overcome potential obstacles prior to the implementation phase. The CNS is prepared to develop and institute educational programs in hospital organizations, ambulatory settings, and community settings alike. This allows for a seamless transition of care from the community and will offer an opportunity to decrease the number of concussions in young adult athletes in the future. 


\section{Nursing \& Healthcare International Journal}

Obstacles, which may be faced when implementing a community based program can include buy in from administrators from the community to include schools, coaches, and facilities where conferences can be held are all important areas that should not be overlooked. It is the needs of the community as well as the community members that should be researched to determine the best possible intervention program that should be developed in order to bring optimal outcomes for all those in a community.

\section{Conclusion}

In summary, a trauma prevention concussion education program targeting the community may be presented with potential challenges or barriers to include: coach and school administrative buy in, time constraints for presenting the conference during preseason, difficulty working within a large group, conflicting ideas, and contractual agreements for specified areas where conferences may be presented. The CNS, as a facilitator of change, has the ability to bring change to fruition and implement a multidisciplinary effort for quality process change to occur. The role of the CNS has been most notably associated with process change, which occurs in the hospital setting; however, in this instance the CNS is stepping outside the box to include the community for public health awareness on trauma prevention of concussions.

\section{References}

1. McGuire CS, McCambridge TM (2011) Concussion in the young athlete: Diagnosis, management, and prevention. Contemporary Pediatrics 28(5): 30-46.

2. Gilchrist J, Thomas KE, Wald M, Langlois J (2007) Nonfatal traumatic brain injuries from sports and recreation activities - United States, 2001-2005. Morbidity and Mortality Weekly 56(29): 733-737.

3. West TA, Bergman K, Biggins MS, French B, Galletly J, et al. (2011) Care of the patient with mild traumatic brain injury. AANN and ARN Clinical Practice Guideline Series: 37.

4. Meehan WP, Zhang J, Mannix R, Whalen MJ (2012) Increasing recovery time between injuries improves cognitive outcome after repetitive mild concussive brain injuries in mice. Neurosurgery 71(4): 885-891.

5. Lincoln AE, Caswell SV, Almquist JL, Dunn RE, Norris JB, et al. (2011) Trends in concussion incidence in high school sports: A prospective 11-year study. Am J Sports Med 39(5): 958-964.

6. Centers for Disease Control (n.d.).

7. California Legislation (2011).

8. Covassin T, Elbin RJ, Sarmiento K (2012) Educating coaches about concussion in sports: evaluation of the CDC's "Heads Up: concussion in youth sports" initiative. J Sch Health 82(5): 233-238.

9. Shenouda C, Hendrickson P, Davenport K, Barber J, Bell KR (2012) The effects of concussion legislation one year later - what have we learned: A descriptive pilot survey of youth soccer player associates. PMR 4(6): 427-435.

10. Vagnozzi R, Signoretti S, Cristofori L, Alessandrini F, Floris R, et al. (2010) Assessment of metabolic brain damage and recovery following mild traumatic brain injury: A multicenter, proton magnetic resonance spectroscopic study in concussed patients. Brain 133(11): 3232-3242.

11. Lear AM, Hoang, MH (2012) Sports concussion: A return to play guide. Journal of Family Practice 61(6): 323-328.

12. Centers for Disease Control (n.d.).

13. Jinguji TM, Bompadre V, Harmon KG, Satchell EK, Gilbert K, et al. (2012) Sport Concussion Assessment Tool-2: baseline values for high school athletes. $\mathrm{Br} \mathrm{J}$ Sports Med 46(5): 365-370.

14. Moser RS, Glatts C, Schatz P (2012) Efficacy of immediate and delayed cognitive and physical rest for treatment of sports-related concussion J Pediatr 161(5): 922-926.

15. Elbin RJ, Schatz P, Covassin T (2011) One-year testretest reliability of the online version of ImPACT in high school athletes. Am J Sports Med 39(11): 23192324.

16. Neal MT, Wilson JL, Hsu W, Powers AK (2012) Concussion: What a neurosurgeon should know about current scientific evidence and management strategies. Surg Neurol Int 3(16).

17. Schatz, P (2010) Long-term test-retest reliability of baseline cognitive assessments using ImPACT. Am J Sports Med 38(1): 47-53. 


\section{Nursing \& Healthcare International Journal}

18. Su JK, Ramirez JF (2012) Management of the athlete with concussion. Perm J 16(2): 54-56.

19. Bader MK, Palmer S, Stalcup C, Shaver T (2002) Using a FOCUS-PDCA quality improvement model for applying the severe traumatic brain injury guidelines to practice: Process and outcomes. Reflect Nurs Leadersh 28(2): 34-35.
20. McCrory P, Meeuwisse W, Johnston K, Dvorak J, Aubry $\mathrm{M}$, et al. (2009) Consensus statement on concussion in sport: The 3rd international conference on concussion in sport held in Zurich, November 2008. Clin J Sport Med 19(3): 185-200. 